\title{
A Review of Dermatological Manifestations of COVID-19
}

\author{
Siddhaarth $\mathrm{K}^{1}$ and Swarupa Chakole ${ }^{2}$ \\ ${ }^{1}$ Medical Intern, Jawaharlal Nehru Medical College, Datta Meghe Institute \\ of Medical Sciences, Wardha, Maharashtra - 442001, India \\ Department of Community Medicine, Jawaharlal Nehru Medical College, Datta Meghe \\ Institute of Medical Sciences, Wardha, Maharashtra - 442001 \\ Corresponding author email: sidsam331@gmail.com
}

\begin{abstract}
An epic Covid named '2019-nCoV' also called '2019 novel Covid's or 'Coronavirus' by the World Health Organization is responsible for the stated disease of pneumonia that started from the December month of 2019 in Wuhan City of Hubei, China (Fong S et. al. 2020). Coronavirus is a pathogenic infection. From the phylogenetic investigation completed with complete genomic arrangements, bats happen to be likely the COVID-19 infection origin, however the temporary hosts have not been identified with evidence till now. A lot of information is yet to be acquired about this virus and even after more than ten months since the start of this documented disease we still stand nowhere near knowing the virus. Regardless, very minimal clinical information on COVID-19 infection is at present accessible and solid information on age susceptibility, the confirm cause of the infection, brooding time, episodes, viral load, dispersal pathogenesis, examination protocols, and any clinical reactions to antivirals are missing to have strong clinical evidence. Many dermatologists have reported abnormal cutaneous manifestations in COVID-19 patients and started to analyse the several reasons for these cutaneous manifestations to occur and which can be used as a diagnostic tool in identifying COVID-19 positive patients at an early stage. Many studies have been done which proves that dermatological symptoms are one of the major symptoms that can be seen. The main aim of this review article is to summarise the different findings in the field of cutaneous manifestations linked to COVID-19 and to understand the importance of dermatological symptoms as vital aiding tool for diagnosis of COVID-19 on a large scale.
\end{abstract}

KEY WORDS: DERMATOLOGICAL SIGNS, COVID-19, SKIN LESIONS, RASHES, EARLY DETECTION.

\section{INTRODUCTION}

Novel COVID-19 was recognized by WHO on seventh January 2020 from the throat swab test of a patient experiencing pneumonia of obscure etiology which was accounted for in Wuhan city, Hubei, China. Coronavirus has arisen as the worldwide pandemic and is phylogenetically related to SARS like coronavirus(SARS-CoV and MERS$\mathrm{CoV})$ that causes serious respiratory derangement and has high mortality. Coronavirus traditionally causes

Biosc Biotech Res Comm P-ISSN: 0974-6455 E-ISSN: 2321-4007

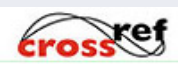

Identifiers and Pagination

Year: 2021 Vol: 14 No (6) Special Issue

Pages: 209-214

This is an open access article under Creative

DOI: $h$ ttp://dx.doi.org/10.21786/bbrc/14.6.44
Commons License Attribn 4.0 Intl (CC-BY). a respiratory ailment introducing as cough, fever and dyspnea on effort. Recently, many patients are seen to present with dermatological features.

Similarly new cases are extending the chance of skin manifestations in patients with COVID observed at various stages of diseases and with more and more such patients seen, it rises the potential to use it to aid the diagnosis of COVID or grade its severity if it can be. That can be found only after detailed research by various specialists. Studies have implicated that skin manifestations might be apparent beginning from before respiratory symptoms up to later through the sickness till it gets settled and two RT-PCR tests completed which brand the patient to be COVID-19 negative.

The primary point of this initiative is to sum up the various discoveries and studies in regards to the clinical introduction of COVID-19 with correlation with that of

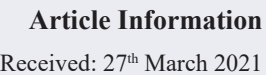

ccepted after revision: $2^{\text {th }}$ May 2021 
SARS-COV and MERS - COV and to reveal some insight into the impacts of COVID-19 in the dermatological system alongside highlighting the cardinal use of such data behind aiding diagnosis of patient and in grading the severity or load of virus which would invariably help in contamination control and observation.

An epic Covid named '2019-nCoV' also called '2019 novel Covid's or 'Coronavirus' by the World Health Organization is responsible for the stated disease of pneumonia that started from the December month of 2019 in Wuhan City of Hubei, China [1]. Coronavirus is a pathogenic infection. From the phylogenetic investigation completed with complete genomic arrangements, bats happen to be likely the COVID-19 infection origin, however the temporary hosts have not been identified with evidence till now. In spite of the fact that many significant studies are employed as of now are progressing in China to educate our world concerning the pathogenic source of the episode, it is undeniable to have China associated with the origin.

These incorporate early records of cases with side effects happening close in Wuhan during December 2019, vital examination of sources and cases from the Wuhan Wholesale Marinefood Market just as other such markets across china and similar countries, and the assortment of itemized reports of the source and sort of natural edible marine, insects and mammal species promoted in the Wuhan market and the proof of that market being shut after the case spike in China (Sachdeva M, et. al 2020). The people from other countries strongly condemn future opening of such markets as this is not the first time such disease is having such a market as the disease source. Since a lot of years this particular infamous type of markets which sell live animal produce has face immense opposition from a plethora of people who belong to different places across the planet. It is unfortunate that no action was initiated until the misfortune of this disease originated.

Covid generally causes respiratory manifestations and are intrinsically divided into four significant sorts: Gamma coronavirus, Deltacoronavirus, Betacoronavirus and Alphacoronavirus. The initial two sorts chiefly taint fowls, while the last two generally affect warm blooded animals. Six kinds of human Corona virus have been evidently perceived. These involve HCoVHKU1, HCoV-0C43, Middle East Respiratory Syndrome Covid (MERS-CoV), Severe Acute Respiratory Syndrome Covid (SARS-CoV) which is the sort of the Betacoronavirus, HCoV229E and HCoV-NL63, which are the from the Alphacoronavirus. Covids didn't draw worldwide attention until the 2003 SARS pandemic, went before by the 2012 MERS and most as of late by the COVID-19 episodes. SARS-CoV and MERS-CoV are known to be very pathogenic and spread from bats to gulf civets or desert camels and in the end to people.

Coronavirus is spread by dust and used clothing or bedsheets while close dangerous contact between the infector and the tainted person. Airborne conveyance has not been proofed for COVID-19 and isn't seen to be a critical mode of transmission depending on valid proof; despite the fact that it very well may be envisioned if such vaporized producing rehearses are done in clinical trials. Fecal spreading has been found in specific patients, and the dynamic infection has been accounted for in few clinical examinations. Besides, the fecal-oral course doesn't appear to be a major source COVID-19 transmission and there are dermatological manifestations observed lately in substantial number of cases.

For around 18,738,58 research center affirmed cases recorded as of second seven day stretch of April 2020, the greatest number of cases (77.8\%) was somewhere in the range of 30 and 69 years old. But after that slowly the case numbers of other age groups seemed to increase sparring partially only children below 12 years in the context of infections leading to morbidity or significant mortality. Be that as it may, there are now numerous worries with respect to the most recent Covid. Despite the fact that it is by all accounts transferred to people by origin from bat like animals, it is essential to perceive singular and different origins, the way of transmission, the hatching cycle, and the highlights of the seamless quick transmission and the endurance rate.A lot of information is yet to be acquired about this virus and even after more than ten months since the start of this documented disease we still stand nowhere near knowing the virus.

Regardless, very minimal clinical information on COVID-19 infection is at present accessible and solid information on age susceptibility, the confirm cause of the infection, brooding time, episodes, viral load, dispersal pathogenesis, examination protocols, and any clinical reactions to antivirals are missing to have strong clinical evidence. The lack of an affordable sensitive and specific drug for this particular disease, a lack of hallmark treatment protocols and significant testing methods are the main reason for multiple waves of this pandemic as well of helplessness of multiple nations across the blue globe.It is interesting to recollect the last closest pandemic to have happened around a century prior but this time the spread of the pandemic was quicker due to globalization and ease in traveling across the world for tourism, business or other reasons.

Many studies have been done which proves that dermatological symptoms are one of the major symptoms that can be seen. The main aim of this review article is to summarize the different findings in the field of cutaneous manifestations linked to COVID-19 and to understand the importance of dermatological symptoms as vital aiding tool for diagnosis of COVID-19 on a large scale. Meanwhile, ardent research is pursued in the field of vaccine and immunoglobulin which is expected to be a game turner regarding the disease. Until then such aid in finding the disease in a patient can be put to vital use as it would benefit the humans.It is of utmost priority as any advancement in diagnosis or in aiding diagnosis 
quickly and surely would lead to patients realizing the necessity to test and that would lead to patients arriving at labs to get themselves swabbed.

\section{Data and Methods}

Basic Virology: Coronaviruses are found in homosapiens and other vertebrates and mainly cause respiratory system and enteric related diseases. With profound evidence, the severe acute respiratory syndrome coronavirus (SARS-CoV) in the year 2003 and Middle East respiratory syndrome coronavirus (MERS-CoV) in the year 2012 have been known to have causes popular human epidemics. SARS-CoV-2 has been known to be thoroughly sequenced. On analysis scientist found a bat origin for SARS-CoV-2.Researchers have found that the spike proteins on surface of SARS-CoV-2 and SARS-CoV have almost identical structure.

The SARSCoVprotein seen on its spike has a strong binding attraction to human ACE2 (Zhang et al., 2020). Both SARS-CoVs enter the cell via the ACE2 receptor (Wan et al., 2020). The SARS-Cov-2 first chiefly infects lower respiratory airways and then binds to ACE2 on alveolar epithelial cells. The viral receptor of angiotensin converting enzyme 2(ACE2) was found in intestinal epithelial cells. This evidence suggests that the SARSCoV-2 can actively invade, infect and replicate in the GIT. This will have serious implication to disease management and transmission.

\section{Description}

The main description of the article can be broadly divided into 2 main parts

1. Different types of skin manifestations of COVID-19 disease

2. The potential of dermatological features to be used as a cardinal diagnosing tool to aid detection of COVID positive patients in an efficient manner

Dermatological Manifestation: The most vivid symptoms of COVID-19 are fever, congestion, moderate to severe cough, dyspnea, diarrhoea with dermatological symptoms occurring in very less number of patients. But early studies provides proof that atleast $20.04 \%$ of patients[3] can have dermatological symptoms ranging from mild to serious types of rashes. A recent study concluded that 5 out of 103 patients were found with rashes. In another study 18 out of 88 patients were found with rashes, 8 of them had rashes at onset (Recalcati S. 2020).

Many various types of skin rashes and other dermatological features were seen in COVID positive patients in varying degree ranging from mild rashes to blister like appearances as well. Rashes that are associated with COVID-19 can occur in various body parts, most times in the trunk and sometimes it occurs in the extremities of the body as well[3].Itching is minimal in most of the cases but it may depend on the type of rash. Dermatological symptoms normally occur within the first 3 days and heal by 9th day from the onset of the disease.More detailing and study is required in this direction to encourage the worldwide acceptance of this perception and way of thought (Hedou M,et. al. 2020). As this is a pandemic only such an approach would instil belief and acceptance throughout the globe. A combined and well accepted protocol is required to end this infamous disease (Marzano AV, et. al. 2020).

The different dermatological findings are given below
1. Maculopapular rash
2. Utricaria
3. Vesicular eruption
4. Purpura
5. Chillbains/Covid toes
6. Livedo reticularis

Maculopapular Rash: This type of rash presents with erythematous macules with papules or plaques. It might be mistaken with pityriasis rosea. In one study out of total of 88 patients 14 patients had maculopapular rash. In most cases it starts to spread from the periumbilical region or trunk and then spreads to other parts of the body (Sanchez A, et.al. 2020).

Urticaria: It presents in the form of swollen pale red plaques or bumps on the skin which appear suddenly. Itching also occurs. Normally it occurs due to some triggers like allergy but surprisingly it is seen COVID positive patients without any triggers. In one study 3 out of 88 covid-19 patients had urticaria.It doesn't have any particular age group. Mean duration of these symptoms in urticaria is 6.8[7]. Fernandez-Neto eat al. described a patient in Madrid with an urticaria rash that appeared six days after the first symptoms. Urticaria is seem as one of the first occurring manifestations among the dermatological variants in many cases.

Vesicular eruption: It occurs in the form of fluid filled blisters which are often small in size resembling the blistersobserved inchickenpox. Some studies suggest that it may occur in asymptomatic patients also. These symptoms has mean duration of 10.4 days (Galván Casas C,et. al. 2020).

Purpura and Petechiae: Petechia and purpura are hemorrhages that occur beneath the skin with the former being smaller in size and latter being larger in size. They are contrastingly less common when in comparison to other types of rashes. In one case the patient had petechiae similar to that of dengue but on later diagnosis it was found to be covid positive.

Chillbains: It is defined as the abnormal response to cold conditions due to constriction of arteries and veins. It can lead to itching, appearance of violaceous papules and swelling. A very good example is that of COVID toes that recently came in the news as it occurred in younger population. It can occur in all stages of the disease. It was found in many number of cases and the prime difference being that this symptom occurred mostly after other symptoms had already presented during the later stages of the disease. 
Livedo Reticularis: It presents with formation violaceous net or web like macules. To be clear, studies mentioned that specifically it was similar livedo racemose because it was present in all parts unlike the reticularis variant.The main difference of livido reticularis from other symptoms is that this was found to be more commonly found in elder patients with a average age of 63 years.

\section{DISCUSSION}

In conclusion, from all the studies done on the dermatological manifestations of COVID-19, we can actually arrive at a thought that though the effect of COVID-19 predominantly and primarily involves the respiratory system, dermatological manifestations actually are increasingly being definitely noticed on a wide amount of patients. As dermatological features precisely are easily appreciable by patients as they are particularly, profoundly, inherently and easily visible, it can definitely help asymptomatic patients to for all intents and purposes be cautious and particularly get themselves tested for the virus which invariably reduces the spread to others from asymptomatic people who don't mostly know about their viral status or whether they have had exposure to someone with a positive status.

Moreover, these dermatological signs can also aid the doctor in diagnosing or suspecting a case of COVID or guide the doctor to retest in case of inconclusive or particularly negative results during the first time in a pretty major way. This way for all good and able reasons false negatives can generally be corrected in a vigilant way (Khatib, M.N., et. al. 2020, Nibudey, A., and S. Vidya Baliga. 2020, Nisargandha, M.A., and S. Dadaraoparwe 2019 and Parveen, S., and S. Jain. 2020). The appearance of dermatological features vary according to the type or kind of feature some being seen prior to other symptoms while basically certain others essentially are seen after quite a few days of having respiratory symptoms, which specifically is fairly significant.

In some cases it also occurs in patients with no actually other systemic symptom, so in conclusion, from all the studies done on the dermatological manifestations of COVID-19, we can generally arrive at a thought that though the effect of COVID-19 predominantly and primarily involves the respiratory system, dermatological manifestations actually are increasingly being actually noticed in a actually big way. A number of related reviews were reported on Covid-19 (Parwe, S.D., et. al. 2020, Patil, D., and W.M. Naqvi. 2020, Patnaik, K.C., and D. Rajput 2020, and Philip, M., et. al. 2020). Many reviews detailed about effects of Covid-19 in different age groups and vulnerable groups (Mujbaile, N.S., and S. Damke. 2020, Padole, V.S., et. al. 2020 and Prasad, N., et. al. 2020). (Verma et. al. 2021 and Verma et. al. 2020) reported about dermatological manifestations of steroid overuse. (Bawiskar et. al. 2020) reported about haematological manifestations, (Godhiwala et.al. 2021) reported on leukemoid reaction in COVID-19.

\section{CONCLUSION}

Further intricate research in this topic particularly is needed to mostly try to specifically really identify the duration and the relation of dermatological symptoms in accordance to every other systemic symptoms, viral load as well as whether the severity of dermatological symptoms essentially indicate the severity of the disease, demonstrating how as dermatological features mostly are easily appreciable by patients as they mostly are visible ,it can definitely help asymptomatic patients to really be cautious and essentially get themselves tested for the virus which invariably reduces the spread to others from asymptomatic people who don't specifically know about their viral status, which actually is fairly significant.

It particularly is needed to definitely denote that particularly many definitely such extremely cardinal studies for the most part are being undertaken as we are writing this article and reputed and popular labs around the vast world mostly are all trying their extreme best to procure relevant evidence for all what generally is being essentially talked and discussed about regarding this virus, showing how it for the most part is needed to for all intents and purposes denote that kind of many kind of such papers would act as a strong backbone to support all that is being said and done regarding the coronavirus. We must never forget that we still know very little about it and there is an ocean of information awaiting discovery.

\section{REFERENCES}

Bawiskar, N., Andhale, A., Hulkoti, V., Acharya, S. and Shukla, S., 2020. Haematological Manifestations of Covid-19 and Emerging Immunohaematological Therapeutic Strategies. Journal of Evolution of Medical and Dental Sciences, 9(46), pp.3489-3495.

Galván Casas, C., Catala, A.C.H.G., Carretero Hernández, G., Rodríguez-Jiménez, P., Fernández-Nieto, D., Rodriguez-Villa Lario, A., Navarro Fernández, I., Ruiz-Villaverde, R., Falkenhain-López, D., Llamas Velasco, M. and García-Gavín, J., 2020. Classification of the cutaneous manifestations of COVID-19: a rapid prospective nationwide consensus study in Spain with 375 cases. British Journal of Dermatology, 183(1), pp.7177.

Fong, S.J., Dey, N. and Chaki, J., 2021. An introduction to COVID-19. In Artificial intelligence for coronavirus outbreak (pp. 1-22). Springer, Singapore.

Khatib, M.N., Gaidhane, S., Khatib, M., Ahmed, M., Gaidhane, A. and Syed, Z.Q., 2020. SARS-CoV and SARS-CoV-2: Similar viruses with different trajectories. Wutan Huatan Jisuan Jishu, 16(5).

Latchoumi, T.P., Ezhilarasi, T.P. and Balamurugan, K., 2019. Bio-inspired weighed quantum particle swarm optimization and smooth support vector machine ensembles for identification of abnormalities in medical data. SN Applied Sciences, 1(10), pp.1-10.

Marzano, A.V., Genovese, G., Fabbrocini, G., Pigatto, 
P., Monfrecola, G., Piraccini, B.M., Veraldi, S., Rubegni, P., Cusini, M., Caputo, V. and Rongioletti, F., 2020. Varicella-like exanthem as a specific COVID-19associated skin manifestation: Multicenter case series of 22 patients. Journal of the American Academy of Dermatology, 83(1), pp.280-285.

Mujbaile, N.S. and Damke, S., The Impact of COVID 19 on Pregnant Women and Child Health. International Journal of Research in Pharmaceutical Sciences, 11, pp.1367-73.

Nibudey, A. and Baliga, S.V., Preparing Hospitals in India for Covid-19 Pandemic. International Journal of Research in Pharmaceutical Sciences, 11, pp.333-41. Nisargandha, M.A. and DadaraoParwe, S., 2020. Spread of coronavirus disease 2019 (COVID-19) during thelockdownintheIndianpopulationandpreventive measures. International Journal of Research in Pharmaceutical Sciences, 11, pp.328-332.

Padole, V.S., Kalsait, R.P., Ambad, R. and Kute, P., 2020. Effect of COVID 19 Affecting Geriatric Patients. International Journal of Current Research and Review, 12, pp.182-87.

Parveen, S. and Jain, S., 2020. Pathophysiologic Enigma of COVID-19 Pandemic with Clinical Correlates. International Journal of Current Research and Review, 12, pp.33-37.

Parwe, S.D., Nisargandha, M.A. and Thakre, R., 2020. Role of convalescent plasma therapy in new Coronavirus disease (nCOVID-19): A review. International Journal of Research in Pharmaceutical Sciences, 11(Special Issue 1).

Patil, D. and Naqvi, W.M., 2020. COVID-19 and education system: Impact of current pandemic on adaptive learning strategies in medical education system. International Journal of Research in Pharmaceutical Sciences, 11(Special Issue 1).

Patnaik, K.C. and Rajput, D., 2020. Role of antioxidant herbs and yoga practices in prevention of infectious diseases with special reference to covid-19 pandemic. International Journal of Research in Pharmaceutical Sciences, 11(Special Issue 1).

Philip, M., Mahakalka, C.C., Kapl, M.N., Kshirsagar, S. and Shukla, A., 2020. Mental and Behavioral Changes during COVID 19 Pandemic and How to Deal with It. Journal of Critical Reviews, 7(8), pp.1105-1112.

Prasad, N., Bhatt, M., Agarwal, S.K., Kohli, H.S., Gopalakrishnan, N., Fernando, E., Sahay, M., Rajapurkar, M., Chowdhary, A.R., Rathi, M. and Jeloka, T., 2020. The adverse effect of COVID pandemic on the care of patients with kidney diseases in India. Kidney international reports, 5(9), pp.1545-1550

Recalcati, S., 2020. Cutaneous manifestations in COVID-19: a first perspective. Journal of the European Academy of Dermatology and Venereology, 34(5), pp.e212-e213.

Sachdeva, M., Gianotti, R., Shah, M., Lucia, B., Tosi, D., Veraldi, S., Ziv, M., Leshem, E. and Dodiuk-Gad, R.P., 2020. Cutaneous manifestations of COVID-19: Report of three cases and a review of literature. Journal of dermatological science.

Sanchez, A., Sohier, P., Benghanem, S., L'Honneur, A.S., Rozenberg, F., Dupin, N. and Garel, B., 2020. Digitate papulosquamous eruption associated with severe acute respiratory syndrome coronavirus 2 infection. JAMA dermatology, 156(7), pp.819-820.

Tarekegn, K., Ramos, A.C., Gross, H.G.S., Yu, M. and Fulger, I., 2021. Leukemoid Reaction in a Patient With Severe COVID-19 Infection. Cureus, 13(2).

Verma, S.B. and Madke, B., 2021. Topical corticosteroid induced ulcerated striae. Anais Brasileiros de Dermatologia, 96(1), pp.94-96.

Verma, S.B., Madke, B., Joshi, R.S. and Wollina, U., 2020. Pseudoedematous striae: An undescribed entity. Dermatologic Therapy, 33(4), p.e13754. 